\title{
Utilidad de la educación en asma bronquial infantil: experiencia piloto
}

\author{
ANA MARÍA HERRERA G.*, AGUSTÍN LEÓN C.*, CARLOS UBILLA P.*, \\ MARÍA ANGÉLICA PÉREZ H.* y JAIME LOZANO C.*
}

Importance of education in pediatric bronchial asthma: pilot experience

Introduction: Even though asthmatic children in Chile have a guaranteed access to treatment, an important number of them have a severe adverse evolution, requiring hospitalization. Education in bronchial asthma is fundamental in the management of these patients, just as pharmacological therapy, as yet most Health Centers in our country do not hold educational activities. Patients and Methods: A pilot prospective study was carried out to evaluate the usefulness of bronchial asthma education, in a group of 12 patients hospitalized for asthma crisis in Clínica Santa María, Santiago de Chile. Patients were randomized in 2 groups, 7 patients receiving formal educational activities and 5 patients receiving the standard information given by their physician. Patients were followed 2, 6 and 12 months after discharge. Results: All studied patients exhibited an improvement in asthma control measured by Asthma Control Test-ACT-, as well as an improvement in quality of life, measured by a questionnaire (PaQLQ). Nonetheless, patients that received the educational activities did attend the Emergency Room less frequently and required less systemic corticosteroid treatments during the study than the patients that did not undergo formal education on asthma. Conclusions: This pilot study suggests that asthma education is a useful tool for patients, in order to achieve a better control of the disease.

Key words: Asthma, education, quality of life, asthma control.

\section{Resumen}

Introducción: A pesar que hoy en día, en Chile, todos los niños asmáticos tienen acceso garantizado al tratamiento, un número importante de ellos evoluciona en forma severa requiriendo hospitalización. La educación en asma bronquial es un pilar fundamental en el manejo de estos pacientes, al igual que el tratamiento farmacológico. Sin embargo, la mayoría de los centros asistenciales de nuestro país no contemplan actividades educativas. Pacientes y Método: Se realizó un estudio piloto, prospectivo para evaluar la utilidad de la educación en asma bronquial en un grupo de 12 pacientes hospitalizados por crisis de asma en Clínica Santa María. Los pacientes fueron distribuidos en forma aleatoria en 2 grupos, 7 pacientes recibieron una actividad educativa formal y 5 la información habitual que entrega el médico tratante al momento del alta. Los pacientes fueron seguidos a los 2, 6 y 12 meses post alta. Resultados: Ambos grupos mejoraron el grado de control de la enfermedad medido por el Asthma Control Test (ACT) y la calidad de vida medida por cuestionario PaQLQ. Los pacientes que recibieron la actividad educativa consultaron menos al servicio de urgencia y requirieron menos curas con corticoides sistémicos durante el año de seguimiento en comparación con el grupo que no la recibió. Conclusiones: La educación parece ser una herramienta útil para lograr una mejor evolución en los pacientes asmáticos.

Palabras clave: Asma, educación, calidad de vida, control del asma.

* Clínica Santa María. Santiago de Chile. 


\section{Introducción}

El asma bronquial es la enfermedad crónica más frecuente en la edad pediátrica. De acuerdo al estudio ISAAC la prevalencia en Chile es de $17,9 \%$ en el grupo de 6 a 7 años y de $15,5 \%$ en el de 13 a 14 años ${ }^{1}$. En la actualidad contamos con excelentes terapias con las cuales la gran mayoría de los pacientes logra el control de la enfermedad. A pesar de lo anterior vemos que una proporción importante de ellos tiene deficiente evolución y calidad de vida, lo que conlleva además un importante gasto en salud. Se sabe que uno de los principales factores responsables del mal control de la enfermedad es la deficiente adherencia al tratamiento, muchas veces por desconocimiento de las bases de la enfermedad, la cronicidad que esta implica y del objetivo del tratamiento. Creemos que la educación es un pilar fundamental que puede subsanar en parte los problemas anteriores, logrando un mejor manejo de los pacientes asmáticos. Nuestra hipótesis es que al implementar un programa educativo podremos mejorar el control de la enfermedad y secundariamente la calidad de vida de nuestros pacientes.

\section{Pacientes y Método}

Se realizó un estudio prospectivo en escolares hospitalizados por exacerbación de asma en el Servicio de Pediatría de la Clínica Santa María desde el 1 de noviembre de 2010 al 1 de noviembre de 2011. Previo consentimiento informado se aplicó a los padres un cuestionario elaborado por los autores para determinar el grado de conocimiento acerca de la enfermedad y a los niños un cuestionario de control de asma (ACT para niños de 4 a 11 años $^{2}$ y ACT para niños de 12 y más años $)^{3}$ y cuestionario de calidad de vida de la Dra. Juniper y cols. (PaQLQ) ${ }^{4}$. Previo al alta se distribuyó en forma aleatoria a los pacientes en dos grupos. El primer grupo recibió la información habitual que entrega el médico tratante a sus pacientes cuando los da de alta y el segundo grupo recibió una actividad educativa formal, la cual consistió en una presentación interactiva, en la cual se entregó información acerca de qué es el asma bronquial, el concepto de cronicidad de la enfermedad, los gatillantes de exacerbación de asma, cómo manejar una crisis, la diferencia entre tratamiento de rescate y de mantención y demostración de la técnica inhalatoria correcta. Posteriormente ambos grupos fueron seguidos por un año para determinar si había diferencias en la evolución. Los pacientes fueron citados y controlados en forma ciega en la Unidad de Enfermedades Respiratorias Pediátricas de Clínica Santa María a los 2, 6 y 12 meses post alta. En estas visitas se repitió ACT, PaQLQ y prueba de conocimientos. En el grupo intervenido se reforzaron además los contenidos educativos y la técnica inhalatoria. Se determinó como resultado principal la mejoría en el ACT y PaQLQ y como resultados secundarios las hospitalizaciones, consultas a Servicio de Urgencia y uso de corticoides sistémicos durante el año de seguimiento.

\section{Resultados}

Durante el período de estudio se reclutó a 12 pacientes con una edad media de 10 años (6 a 13 años), 9 de género masculino. La mediana de días de hospitalización fue de 3 días (1 a 7), sólo un paciente requirió manejo en Unidad de Paciente Crítico (UPC). Del total de pacientes 3 tenían antecedentes de consulta a Servicio de Urgencia (1, 3 y 4 veces respectivamente) y uno hospitalizaciones el año previo. Este último paciente registraba 2 ingresos por crisis de asma, una de las cuales se realizó en UPC. Se constató además el uso de corticoides sistémicos durante el año anterior al estudio en $5 / 12$ pacientes ( 1 a 7 cursos). Con respecto a la terapia, la totalidad de los pacientes reclutados tenía indicado el uso de corticoides inhalados permanentes, sin embargo, sólo 3/12 (25\%) lo estaba usando al momento de la hospitalización. En relación a la contaminación intradomiciliaria la mitad de los padres reconoció el hábito tabáquico en domicilio. Sólo 3 niños utilizaban métodos de calefacción no contaminantes (calefacción eléctrica o loza radiante), el resto utilizaba mayoritariamente gas $(\mathrm{n}=5)$, seguido por parafina $(\mathrm{n}=3)$ y leña $(\mathrm{n}=$ 1). En $9 / 12$ niños (75\%) existía el diagnóstico médico de rinitis alérgica, sin embargo, sólo 6 recibían tratamiento regular. Por otro lado 5/12 $(41,6 \%)$ tenían diagnóstico de dermatitis atópica. Del total de pacientes 11 se habían realizado test cutáneo, encontrándose que en $10(90,9 \%)$ había sensibilización al menos a un alergeno y en 7/10 (70\%) polisensibilización. La evaluación nutricional demostró que sólo 2 pacientes eran eutróficos, los 10 restantes $(83,3 \%)$ tenían mal nutrición por exceso, 6 sobrepeso y 4 obesidad. En lo que concierne a la encuesta de conocimientos acerca de la enfermedad la gran mayoría de los padres tenían el concepto de que el asma es una enfermedad de origen alérgico (10/12) y que es crónica (9/12). Cuatro familias señalaron que el objetivo del tratamiento era que el niño dejara 
de ser asmático y en 8 evitar que tenga síntomas. En relación a la racionalidad del tratamiento, 4 familias señalaron que el salbutamol se usaba para prevenir las crisis y otras 4 que los corticoides inhalados eran para tratar las crisis. Cuatro pacientes $(33,3 \%)$ indicaron que usaban el tratamiento controlador sólo cuando tenían síntomas. Por otro lado, 10/12 señalaron que el tratamiento con corticoides inhalados debía ser permanente ya que de lo contrario esto implicaba un riesgo para el paciente, principalmente la aparición de crisis (8/10). En 5 casos los padres respondieron que les daba miedo usar corticoides inhalados, 3 por el efecto de éstos en el crecimiento, uno por pensar que el tratamiento puede ser adictivo y otro simplemente por considerarlo dañino para la salud. En 5 casos (41\%) la administración del tratamiento de mantención era responsabilidad del niño sin supervisión alguna de los padres. Sólo 3 pacientes señalaron tener control periódico con su médico tratante cada 2 a 3 meses, el resto acudía sólo cuando tenía crisis. Dos pacientes no tenían médico tratante, utilizando sólo el Servicio de Urgencia cuando presentaban crisis. Ningún niño realizaba la técnica inhalatoria en forma completamente correcta, a pesar de que todos usaban aerocámara. Muchos niños seguían utilizando la técnica nasobucal a pesar de ser escolares y los tiempos inspiratorios en muchos casos no eran los adecuados. Previamente a la aplicación de la intervención observamos que ambos grupos de pacientes eran bastante similares en cuanto a sus características basales (Tabla 1), sólo la proporción de niños de género masculino fue mayor en el grupo que recibió educación. El control del asma medido por cuestionario ACT y la calidad de vida medida por el cuestionario PaQLQ mejoraron en forma importante en ambos grupos a lo largo del estudio, sobre todo los valores previos al alta y los registrados en los controles posteriores (Tabla 2). Sin embargo, no se logró evidenciar diferencias significativas entre ambos grupos (Tabla 3). En la evaluación de los 6 meses post alta se observó un mal control de la enfermedad definido por un ACT $\leq 19$ en 1 de 7 pacientes en el grupo con educación $(14,2 \%)$ y en 1 de 5 del grupo sin educación $(20 \%)$. Al año post alta se observó que todos los pacientes del grupo con educación

Tabla 1. Características basales de los niños asmáticos con y sin educación

\begin{tabular}{|lcc|}
\hline & Grupo con educación & Grupo sin educación \\
$\mathrm{n}$ & 7 & 5 \\
Edad promedio (rango), años & $10(6$ a 13) & $9(6$ a 12$)$ \\
Género masculino (n) & 6 & 3 \\
ACT $\leq 19$ puntos (n) & 4 & 3 \\
PaQLQ $\leq 5$ puntos (n) & 4 & 4 \\
Tratamiento con corticoides inhalados (n) & 5 & 4 \\
\hline
\end{tabular}

Tabla 2. Puntajes promedio de los cuestionarios de control de asma $\mathrm{ACT}^{2,3}$ y de calidad de vida $\operatorname{PaQLQ}^{4}$ de los 12 niños enrolados durante el estudio

\begin{tabular}{|ccccc|}
\hline Cuestionario & Previo alta & 2 meses & 6 meses & 1 año \\
ACT & 18,8 & 23,1 & 23,3 & 23,6 \\
PaQLQ & 4,6 & 6,2 & 6,5 & 6,6 \\
\hline
\end{tabular}

Tabla 3. Puntajes promedio de los cuestionarios $\mathrm{ACT}^{2,3}$ y $\operatorname{PaQLQ}^{4}$ de niños asmáticos con y sin educación durante el estudio

\begin{tabular}{|lcccccc|}
\hline & \multicolumn{2}{c}{ 2 meses } & \multicolumn{2}{c}{ 6 meses } & \multicolumn{2}{c|}{12 meses } \\
Cuestionario & ACT & PaQLQ & ACT & PaQLQ & ACT & PaQLQ \\
Con educación $(\mathrm{n}=7)$ & 24,1 & 6,5 & 23,7 & 6,5 & 24,7 & 6,7 \\
Sin educación $(\mathrm{n}=5)$ & 21,8 & 5,8 & 22,8 & 6,5 & 22,2 & 6,5 \\
\hline
\end{tabular}


Tabla 4. Número de niños asmáticos que necesitaron hospitalización, consulta de urgencia o cura corticoidal durante el período del estudio

\begin{tabular}{|lccc|}
\hline & Hospitalización & Servicio de Urgencia & Cura corticoidal \\
Con educación $(\mathrm{n}=7)$ & 0 & 0 & 1 \\
Sin educación $(\mathrm{n}=5)$ & 1 & 2 & 5 \\
\hline
\end{tabular}

(7/7) tenían ACT $\geq 19$ en comparación con 4/5 del grupo sin educación. Durante el período de estudio sólo un paciente vuelve a hospitalizarse, 3 meses luego del primer ingreso. Este paciente pertenecía al grupo sin educación. Dos pacientes debieron acudir al Servicio de Urgencia por crisis de asma, ambos del grupo sin educación. Durante el período de estudio 6 pacientes requirieron curas con corticoides sistémicos 5 del grupo control y 1 del grupo intervenido (Tabla 4).

\section{Discusión}

En la actualidad contamos con excelentes terapias para el manejo del asma bronquial, a pesar de lo cual el control de la enfermedad no es adecuado, lo que se traduce en un aumento de la morbilidad, como lo pudimos evidenciar en nuestros pacientes. Durante el año previo a la hospitalización $25 \%$ de los niños debió acudir al Servicio de Urgencia por crisis de asma y $41 \%$ debió utilizar corticoides sistémicos por el mismo motivo. Previo al alta pudimos observar además un mal control de la enfermedad medido por ACT en $58,3 \%$ de los casos. En un estudio realizado en Estados Unidos en 2.429 niños asmáticos de 4 a 17 años se observó que el asma no controlado medido por ACT correspondía a un $46 \% \%^{5}$. A nivel latinoamericano el estudio AIRLA ${ }^{6}$ también nos muestra cifras desalentadoras. En 808 asmáticos menores de 16 años de 11 países se observó que en los últimos 12 meses, $57 \%$ presentaba síntomas diurnos, 47\% síntomas nocturnos y $69 \%$ consultas a Servicio de Urgencia. A nivel nacional, en un trabajo realizado en adolescentes asmáticos, se evidenció un mal control de la enfermedad en $46,3 \%$ de los casos ${ }^{7}$. Se sabe además que la falta de control conduce a una mala calidad de vida como lo demostró una investigación en escolares chilenos, en la que se observó mala calidad de vida en cerca de $40 \%$ de los niños y en $68 \%$ de sus cuidadores ${ }^{8}$. En nuestros pacientes la calidad de vida promedio evaluada por PaQLQ fue de 4,6 con un $66,6 \%$ de los niños con puntaje bajo 5 . Las causas de mal control en los pacientes con diagnóstico confirmado de asma son varia- das, una de ellas es la presencia de comorbilidades no tratadas ${ }^{9,10}$. En nuestros pacientes pudimos comprobar la presencia de rinitis alérgica, la cual estaba siendo manejada sólo en un tercio de los casos. Otra causa de falta de control es la exposición a agentes ambientales, principalmente humo del cigarrillo, lo que pudimos observar en la mitad de nuestros pacientes. Este es un punto importante a tener en consideración ya que los niños asmáticos expuestos al humo del cigarrillo tienen 4 veces más probabilidades de tener mal control de la enfermedad ${ }^{11}$ y por otro lado en adultos asmáticos fumadores se ha demostrado resistencia al tratamiento con corticoides inha$\operatorname{lados}^{10}$. Otra causa de falta de control es una técnica inhalatoria deficiente, lo que estaba presente en mayor o menor grado en la totalidad de nuestros niños. Al respecto se ha demostrado que el control empeora en la medida que aumenta el número de errores en la técnica inhalatoria ${ }^{10}$. Sin embargo, la principal causa de falta de control del asma bronquial es la falta de adherencia al tratamiento ${ }^{12,13}$. Esto lo pudimos observar en nuestro estudio ya que sólo $25 \%$ de los niños estaba usando el tratamiento controlador al momento de la hospitalización. Esta realidad contrasta con el hecho de que en la encuesta realizada a los padres un $83,3 \%$ contestó que el tratamiento con corticoides inhalados debía ser utilizado en forma permanente. Algo similar se evidenció a nivel nacional en otro estudio realizado en 67 niños asmáticos hospitalizados por crisis de asma en UPC. La mala adherencia al tratamiento en éstos llegó al $74 \%{ }^{14}$. La falta de adherencia es un problema central, que conduce a un mal control de la enfermedad con aumento de las exacerbaciones y aumento del riesgo de mortalidad. Al respecto en un trabajo de investigación se observó que los niños que tenían crisis de asma y que requerían del uso de corticoides sistémicos tenían una adherencia al tratamiento de mantención de $13,7 \%$ en comparación con $68,2 \%$ en los que no tuvieron exacerbación ${ }^{15}$. En este mismo estudio se evidenció la sobrevaloración de la adherencia medida por cuestionario en comparación con la adherencia medida electrónicamente, 95,4\% versus $58,4 \%$. Un resultado similar es reportado 
por otra publicación, donde además se comprobó una disminución progresiva de la adherencia en el tiempo. Al comienzo la adherencia referida por los padres era de $100 \%$ comparada con $58,3 \%$ del dispositivo electrónico y al cabo de 12 meses de seguimiento la primera era de $96,4 \%$ y la segunda de tan sólo $34,9 \%{ }^{16}$. Se estima que una adherencia adecuada al tratamiento se logra cuando el medicamento es usado $80 \%$ o más de lo indicado. Desgraciadamente la literatura demuestra que la adherencia al tratamiento en pacientes asmáticos es baja, generalmente bajo $50 \%{ }^{17}$. Se sabe que la falta de adherencia en los pacientes asmáticos puede ser de dos tipos, no intencional o intencional ${ }^{18}$. En nuestros pacientes pudimos ver reflejados varios problemas de carácter no intencional como falta de entendimiento del régimen de tratamiento, lo que explicaría que algunos pacientes usaran el broncodilatador en forma permanente y el corticoide inhalado sólo para las crisis. En este grupo también evidenciamos mala adherencia intencional ya que la gran mayoría de los padres teóricamente sabía que el tratamiento con corticoides inhalados debía ser administrado en forma permanente; sin embargo, en la práctica no lo utilizaban, principalmente por temor a los potenciales efectos adversos. Teniendo en cuenta que la principal causa de falta de control en los asmáticos es la falta de adherencia al tratamiento se entiende la gran importancia que cumple la educación $^{12,13}$. El beneficio de las actividades educativas ha sido demostrado en varios estudios, observándose mejoría de la función pulmonar, reducción del ausentismo escolar, disminución del número de días sin actividad física, disminución del número de consultas a Servicios de Urgencia, mejoría del control medido por ACT y mejoría de la adherencia al tratamiento de mantención entre otros ${ }^{19-21}$. A nivel nacional también se observó que una actividad educativa en escolares asmáticos lograba una mejoría significativa del control del asma medido por cuestionario CAN a los 4 meses de realizada ${ }^{22}$. En nuestro estudio quisimos comparar la evolución de un grupo de asmáticos hospitalizados que recibió una actividad educativa formal versus un grupo control. Pudimos observar a lo largo del seguimiento que ambos grupos mejoraron en forma significativa el control del asma medido por ACT y la calidad de vida medida por PaQLQ, sin diferencias significativas entre ambos. Esto se podría explicar porque el grupo sin educación también fue evaluado a los 2, 6 y 12 meses post alta y está demostrado que el solo hecho de citar en forma regular al paciente se asocia a mejoría de la adherencia y del control ${ }^{23,24}$. Otra explicación posible sería que dado el bajo número de pacientes enrolados en el estudio no se logró alcanzar poder estadístico para observar una diferencia entre ambos grupos. Con respecto a los resultados secundarios observamos que los niños que recibieron la actividad educativa consultaron menos al Servicio de Urgencia y utilizaron menos corticoides sistémicos durante el año de seguimiento en comparación con los niños que no la recibieron, aunque esta diferencia no alcanzó significación estadística.

Para finalizar debemos decir que la fortaleza del presente estudio de educación en asma bronquial es que es el primero en nuestro medio en ser prospectivo y aleatorio y que su debilidad es el escaso número de pacientes enrolados. A pesar que el número de niños en este estudio piloto es pequeño, creemos que los resultados marcan una tendencia en favor del empleo de la educación en asma bronquial y esperamos que una serie con mayor número de pacientes pueda validar este hallazgo. A nivel asistencial hay poco tiempo para hacer educación al momento de atender al paciente, por lo que esta actividad debiera ser realizada en una instancia independiente. Al momento de realizarla es importante abarcar todos los puntos que ésta contempla: entregar información en un lenguaje que sea entendible para el paciente, derribar mitos, enseñar la técnica inhalatoria correcta, entregar herramientas de automonitoreo, un adecuado plan de acción a la medida del paciente y contemplar controles regulares ${ }^{25}$. El gasto de implementar una actividad educativa de este tipo se justifica plenamente ya que ha demostrado ser costo efectiva ${ }^{26}$. Por todo lo expuesto pensamos que la educación en asma bronquial debiera ser incorporada en forma rutinaria al manejo de todos nuestros pacientes.

\section{Bibliografía}

1.- MALlOL J, AGUiRRE V, AGUILAR P, CALVO M, AMARALES L, ARELLANO P, et al. Cambios en la prevalencia de asma en escolares chilenos entre 1994 y 2002: International Study of Asthma and Allergies in Childhood (ISAAC) - Chile phases I and III. Rev Med Chile 2007; 135: 580-6.

2.- www.asthmacontroltest.com/countries/spain04/page two.asp? country $=$ SPAIN\&lang $=$.

3.- www.allergyassoc.net/337-asthma-control-test-child4-11-spanish-version1.pdf

4.- JUNIPER E F, GUYATT G H, FEENY D H, FERRIE P J, GRIFFITH L E, TOWNSEND M. Measuring quality of life in children with asthma. Qual Life Res 1996; 5: 35-46.

5.- $\quad$ LIU A H, GILSENAN A W, STANFORD R H, LINCOURT W, ZIEMIECKI R J, ORTEGA H. Status of 
asthma control in pediatric asthma control characteristics and prevalence survey study (ACCESS). Pediatr 2010; 157: 276-81.

6.- NEFFEN H, FRITSCHER C, CUEVAS F, LEVY G, CHIARELLA P, SORIANO J B, et al. Asthma control in Latin America: the asthma insights and reality in Latin America (AIRLA) survey. Rev Panam Salud Pública 2005; 17: 191-7.

7.- VIDAL A, UBILlA C, DUFFAU G. Control de asma en adolescentes. Rev Med Chile 2008; 136: 859-66.

8.- VIDAL A, DUFFAU G, UBILLA C. Calidad de vida en el niño asmático y su cuidador. Rev Chil Enf Respir 2007; 23: 160-6.

9.- LØDRUP CARLSEN K C, HEDLIN G, BUSH A, WENNERGREN G, DE BENEDICTIS F M, DE JONGSTE J C, et al. Assessment of problematic severe asthmain children. Eur Respir J 2011; 37: 432-40.

10.- HAUGHNEY J, PRICE D, KAPLAN A, CHRYSTYN H, HORNE R, MAY N, et al. Achieving asthma control in practice: Understanding the reasons for poor control. Respiratory Medicine 2008; 102: 1681-93.

11.- MCGHAN S L, MACDONALD C, JAMES D E, NAIDU P, WONG E, SHARPE H, et al. Factors associated with poor asthma control in children aged five to 13 years. Can Respir J 2006; 13: 23-9.

12.- BOURDIN A, HALIMI L, VACHIER I, PAGANIN F, LAMOUROUX A, GOUITAA M, et al. Adherence in severe asthma. Clin Exp. Allergy 2012; 42: 1566-74.

13.- BUSH A, SAGLANI S. Management of severe asthma in children. Lancet 2010; 376: 814-25.

14.- HERRERA A M, ESCOBAR A M, SAAVEDRA M, MEDINA M E, SALGADO E, PALAVECINO, et al. Exacerbación de asma infantil en unidad de paciente crítico: caracterización clínica. Rev Chil Enf Respir 2011; 27: 26-30.

15.- MILGROM H, BENDER B, ACKERSON L, BOWRY P, SMITH B, RAND C. Noncompliance and treatment failure in children with asthma. J Allergy Clin Immunol 1996; 98: 1051-7.

16.- JENTZSCH N S, CAMARGOS P A M, COLOSIMO E A, BOUSQUET J. Monitoring adherence to beclomethasone in asthmatic children and adolescents through four different methods. Allergy 2009; 64: 145862.

17.- DESAI M, OPPENHEIMER J J. Medication adherence in the asthmatic child. Curr Allergy Asthma Rep 2011; 11: 454-64.

18.- RAU J L. Determinants of Patient Adherence to an Aerosol Regimen. Respir Care 2005; 50: 1346-56.

19.- GUEVARA J P, WOLF F M, GRUM C M, CLARK N $M$. Effects of educational interventions for self management of asthma in children and adolescents: systematic review and meta-analysis. Brit Med J 2003; 326: 13089.

20.- PARK J, JACKSON J, SKINNER E, RANGHELL K, SAIERS J, CHERNEY B. Impact of an Adherence Intervention Program on Medication Adherence Barriers, Asthma Control, and Productivity/Daily Activities in Patients With Asthma. J Asthma 2010; 47: 1072-7.

21.- DALCIN PDE T, GRUTCKI D M, LAPORTE P P, LIMA P B, VIANA P V, KONZEN G L, et al. Impact of a short-term educational intervention on adherence to asthma treatment and on asthma control. J Bras Pneumol 2011; 37: 19-27.

22.- HENRÍQUEZ T, CERUTI E. Educación en niños asmáticos controlados en hospital Roberto del Río. Impacto en conocimientos, adherencia y control de la enfermedad. Rev Chil Enf Respir 2013; 29: 70-4.

23.- BURGESS S, SLY P D, DEVADASON S. Providing Feedback on Adherence Increases Use of Preventive Medication by Asthmatic Children. Journal of Asthma 2010; 47: 198-201.

24.- BENDER B, MILGROM H, APTER A. Adherence intervention research: What have we learned and what do we do next? J Allergy Clin Immunol 2003; 112: 489-94.

25.- BROUWER A, BRAND P. Asthma education and monitoring: what has been shown to work. Paediatr Respir Rev 2008; 9: 193-200.

26.- KAMPS A W A, ROORDA R J, KIMPEN J L L, OVERGOOR-VAN DE GROES A W, VAN HELSDINGEN-PEEK LCJAM, et al. Impact of nurse-led outpatient management of children with asthma on healthcare resource utilisation and costs. Eur Respir J 2004; 23: 304-9.

Correspondencia a:

Dra. Ana María Herrera G.

Clinica Santa María

Email: amherreragana@gmail.com 